\title{
Deux modèles effectifs pour les champs électromagnétiques dans le vide de Dirac
}

\author{
Philippe Gravejat, Christian Hainzl, Mathieu Lewin ${ }^{3}$ et Éric Séré ${ }^{4}$
}

31 août 2016

\begin{abstract}
Résumé
Cet exposé présente des résultats récents [17, 18] quant à deux modèles effectifs pour les effets de la polarisation du vide quantique sur les champs électromagnétiques. Le modèle de Pauli-Villars les décrit de manière rigoureuse lorsque les champs électromagnétiques sont supposés classiques. À partir de ce premier modèle est ensuite proposée une dérivation du modèle classique d'Euler-Heisenberg [22] dans un régime de champs purement magnétiques et faiblement variables.
\end{abstract}

\section{Introduction}

Le vide quantique présente des fluctuations d'énergie qui se manifestent expérimentalement par des phénomènes tels que l'effet Casimir [4, 5] ou le décalage de Lamb [23]. Ces fluctuations d'énergie induisent la production de paires électron-positron virtuelles qui se conduisent comme des dipôles électriques et interagissent avec les champs électromagnétiques. Le vide quantique se comporte ainsi comme un milieu polarisable [14, 15, 22, 33]. Ce sont ces influences réciproques entre le vide quantique et les champs électromagnétiques que nous allons tâcher de mieux comprendre à travers l'analyse mathématique de deux modèles simples.

Avant d'introduire plus en détail ces deux modèles, revenons un instant à la description classique des champs électromagnétiques, qui repose sur les équations de Maxwell. Dans le cas stationnaire qui nous intéresse dans la suite, ces équations se réduisent aux équations de Poisson pour les potentiels électrique $V_{\text {clas }}$ et magnétique $A_{\text {clas }}$ dans la jauge de Coulomb

$$
\operatorname{div} A_{\text {clas }}=0 .
$$

Dans des unités physiques ad hoc et en présence de densités externes de charge $\rho_{\text {ext }}$ et de courant $j_{\text {ext }}$, ces potentiels satisfont le système

$$
\left\{\begin{array}{l}
-\Delta V_{\text {clas }}=4 \pi e \rho_{\text {ext }}, \\
-\Delta A_{\text {clas }}=4 \pi e j_{\text {ext }},
\end{array}\right.
$$

1. Université de Cergy-Pontoise, Laboratoire de Mathématiques Analyse, Géométrie, Modélisation (UMR 8088), 95302 Cergy-Pontoise cedex, France. E-mail : philippe.gravejat@u-cergy.fr

2. Universität Tübingen, Mathematisches Institut, Auf der Morgenstelle 10, 72076 Tübingen, Germany. E-mail : christian.hainzl@uni-tuebingen.de

3. CNRS et Université Paris-Dauphine, Centre de Recherche en Mathématiques de la Décision (UMR 7534), Place du Maréchal de Lattre de Tassigny, 75775 Paris cedex 16, France. E-mail : Mathieu.Lewin@math.cnrs.fr

4. Université Paris-Dauphine, Centre de Recherche en Mathématiques de la Décision (UMR 7534), Place du Maréchal de Lattre de Tassigny, 75775 Paris cedex 16, France. E-mail : sere@ceremade.dauphine.fr 
dans lequel $e$ désigne la charge élémentaire d'un électron. Les champs électrique $E_{\text {clas }}$ et magnétique $B_{\text {clas }}$ sont alors donnés par les formules

$$
E_{\text {clas }}:=-\nabla V_{\text {clas }}, \quad \text { et } \quad B_{\text {clas }}:=\operatorname{rot} A_{\text {clas }} .
$$

Les équations de Poisson (2) s'interprètent comme celles de points critiques de l'action lagrangienne classique $\mathcal{L}_{\text {clas }}$ définie par la formule

$$
\mathcal{L}_{\text {clas }}(\boldsymbol{A}):=\frac{1}{8 \pi} \int_{\mathbb{R}^{3}}\left(|E|^{2}-|B|^{2}\right)-e \int_{\mathbb{R}^{3}}\left(\rho_{\text {ext }} V-j_{\text {ext }} \cdot A\right),
$$

dans laquelle nous avons introduit le potentiel électromagnétique $\boldsymbol{A}:=(V, A)$. L'unique potentiel électromagnétique $\boldsymbol{A}_{\text {clas }}$ qui satisfait le système (2) dans la jauge de Coulomb (1) s'obtient comme l'unique solution du problème de min-max

$$
\mathcal{L}_{\text {clas }}\left(\boldsymbol{A}_{\text {clas }}\right)=\min _{V \in \dot{H}^{1}\left(\mathbb{R}^{3}\right)} \max _{A \in \dot{H}_{\text {div }}^{1}\left(\mathbb{R}^{3}\right)} \mathcal{L}_{\text {clas }}(\boldsymbol{A})\left(=\max _{A \in \dot{H}_{\text {div }}^{1}\left(\mathbb{R}^{3}\right)} \min _{V \in \dot{H}^{1}\left(\mathbb{R}^{3}\right)} \mathcal{L}_{\text {clas }}(\boldsymbol{A})\right) .
$$

Les espaces de Sobolev homogènes $\dot{H}^{1}\left(\mathbb{R}^{3}\right)$ et $\dot{H}_{\text {div }}^{1}\left(\mathbb{R}^{3}\right)$ sont ici définis par les expressions

$$
\dot{H}^{1}\left(\mathbb{R}^{3}\right):=\left\{f \in L^{6}\left(\mathbb{R}^{3}, \mathbb{R}\right) \text {, t.q. } \nabla f \in L^{2}\left(\mathbb{R}^{3}, \mathbb{R}\right)^{3}\right\},
$$

et

$$
\dot{H}_{\text {div }}^{1}\left(\mathbb{R}^{3}\right):=\left\{g \in \dot{H}^{1}\left(\mathbb{R}^{3}\right)^{3}, \text { t.q. } \operatorname{div} g=0\right\} .
$$

Revenons maintenant aux effets de la polarisation du vide quantique sur le champ électromagnétique. La description lagrangienne précédente autorise une prise en compte très simple de ces effets. Il suffit d'ajouter à la définition de l'action lagrangienne un terme qui traduit les interactions entre le vide quantique et le champ électromagnétique. Cette idée conduit à l'introduction d'une action lagrangienne effective de la forme

$$
\mathcal{L}_{\text {eff }}(\boldsymbol{A}):=-\mathcal{F}_{\text {vac }}(e \boldsymbol{A})+\frac{1}{8 \pi} \int_{\mathbb{R}^{3}}\left(|E|^{2}-|B|^{2}\right)-e \int_{\mathbb{R}^{3}}\left(\rho_{\mathrm{ext}} V-j_{\mathrm{ext}} \cdot A\right) .
$$

Dans le cas stationnaire, le champ électromagnétique $\boldsymbol{F}_{\text {eff }}:=\left(E_{\text {eff }}, B_{\text {eff }}\right)$ est associé comme avant à un point critique $\boldsymbol{A}_{\text {eff }}$ de l'action effective $\mathcal{L}_{\text {eff }}$. Ce point critique satisfait des équations de Poisson

$$
\left\{\begin{array}{l}
-\Delta V_{\mathrm{eff}}=4 \pi e\left(\rho_{\mathrm{vac}}\left(e \boldsymbol{A}_{\mathrm{eff}}\right)+\rho_{\mathrm{ext}}\right), \\
-\Delta A_{\mathrm{eff}}=4 \pi e\left(j_{\mathrm{vac}}\left(e \boldsymbol{A}_{\mathrm{eff}}\right)+j_{\mathrm{ext}}\right),
\end{array}\right.
$$

dans la jauge de Coulomb (1). Les quantités $\rho_{\text {vac }}\left(e \boldsymbol{A}_{\text {eff }}\right)$ et $j_{\text {vac }}\left(e \boldsymbol{A}_{\text {eff }}\right)$ sont données, au moins formellement, par les formules

$$
e \rho_{\mathrm{vac}}\left(\boldsymbol{A}_{\mathrm{eff}}\right):=\frac{\partial \mathcal{F}_{\mathrm{vac}}}{\partial V}\left(e \boldsymbol{A}_{\mathrm{eff}}\right), \quad \text { et } \quad e j_{\mathrm{vac}}\left(\boldsymbol{A}_{\mathrm{eff}}\right):=-\frac{\partial \mathcal{F}_{\mathrm{vac}}}{\partial A}\left(e \boldsymbol{A}_{\mathrm{eff}}\right) .
$$

Elles s'interprètent comme les densités de charge et de courant du vide quantique en présence d'un potentiel électromagnétique $\boldsymbol{A}_{\text {eff }}$.

Ces densités de charge et de courant apportent des corrections non linéaires au calcul du champ électromagnétique. Elles sont négligeables dans des conditions expérimentales normales, mais sont supposées jouer un rôle notable au dessus d'une valeur critique du champ électromagnétique, appelée limite de Schwinger. De tels champs sont présumés atteints dans les magnétoiles, étoiles à neutrons munies d'un champ magnétique très fort $[2$, 
$27,7]$. La détection des effets non linéaires de ces densités de charge et de courant est un sujet de recherche particulièrement actif $[3,28]$.

Toute la difficulté de cette description lagrangienne de la polarisation du vide réside dans le calcul de la fonctionnelle $\mathcal{F}_{\text {vac }}(e \boldsymbol{A})$. Dans le cas stationnaire qui nous intéresse ici, il est naturel que ce terme corresponde à l'énergie fondamentale du vide quantique en présence du potentiel électromagnétique $\boldsymbol{A}$. Il s'avère toutefois délicat de donner une valeur définie à ce terme en raison des divergences bien connues qui apparaissent dans sa définition.

Euler et Heisenberg [22] (cf. aussi Weisskopf [33]) ont néanmoins calculé la valeur de l'énergie du vide $\mathcal{F}_{\text {vac }}(e \boldsymbol{A})$ dans la limite des champs électromagnétiques faiblement variables. L'énergie dite d'Euler-Heisenberg qu'ils ont déterminée est donnée par l'expression

$$
\begin{aligned}
\mathcal{F}_{\mathrm{vac}}^{\mathrm{EH}}(e \boldsymbol{A}):=\frac{1}{8 \pi^{2}} \int_{\mathbb{R}^{3}} \int_{0}^{\infty} d s \frac{e^{-s m^{2}}}{s^{3}}\left(\frac{e^{2} s^{2}}{3}\right. & \left(|E|^{2}-|B|^{2}\right)-1+e^{2} s^{2}(E \cdot B) \times \\
& \left.\times \frac{\operatorname{Re} \cosh \left(e s\left(|B|^{2}-|E|^{2}+2 i E \cdot B\right)^{\frac{1}{2}}\right)}{\operatorname{Im} \cosh \left(e s\left(|B|^{2}-|E|^{2}+2 i E \cdot B\right)^{\frac{1}{2}}\right)}\right) .
\end{aligned}
$$

Cette expression n'est pas toujours bien définie. Lorsque le champ électromagnétique ne satisfait pas les contraintes $E \cdot B=0$ et $|E|<|B|$, l'intégrande ci-dessus présente des pôles. Une façon classique de donner un sens rigoureux à cette énergie consiste à remplacer la variable d'intégration $s$ par une variable complexe $s+i \eta$, puis à prendre la limite $\eta \rightarrow 0$. La fonction ainsi obtenue peut présenter une partie imaginaire non nulle, qui s'interprète comme le taux de production de paires électron-positron [19, Paragraphe 7.3], et rend donc compte des fluctuations d'énergie du vide quantique.

Dans le cas purement magnétique où le champ électrique $E$ est identiquement nul, l'énergie d'Euler-Heisenberg est bien définie par la formule

$$
\mathcal{F}_{\text {vac }}^{\mathrm{EH}}(e A)=\int_{\mathbb{R}^{3}} f_{\text {vac }}^{\mathrm{EH}}(e B):=\frac{1}{8 \pi^{2}} \int_{\mathbb{R}^{3}} \int_{0}^{\infty} d s \frac{e^{-s m^{2}}}{s^{3}}\left(e s|B| \operatorname{coth}(e s|B|)-1-\frac{e^{2} s^{2}|B|^{2}}{3}\right) .
$$

La densité d'énergie par unité de volume $f_{\text {vac }}^{\mathrm{EH}}$ est négative, décroissante et concave, ce qui signifie que la polarisation du vide tend à amplifier la valeur du champ magnétique, et non à l'écranter. Cette densité se comporte comme

$$
f_{\text {vac }}^{\mathrm{EH}}(e B) \sim-\frac{e^{4}}{360 \pi^{2} m^{4}}|B|^{4},
$$

lorsque le champ magnétique $|B| \rightarrow 0$, et

$$
f_{\mathrm{vac}}^{\mathrm{EH}}(e B) \sim-\frac{e^{2}|B|^{2}}{24 \pi^{2}} \ln \left(\frac{e|B|}{m^{2}}\right),
$$

lorsque $|B| \rightarrow+\infty$. L'action lagrangienne correspondante

$$
\mathcal{L}_{\text {eff }}^{\mathrm{EH}}(A):=-\int_{\mathbb{R}^{3}} f_{\mathrm{vac}}^{\mathrm{EH}}(e B)-\frac{1}{8 \pi} \int_{\mathbb{R}^{3}}|B|^{2}+e \int_{\mathbb{R}^{3}} j_{\mathrm{ext}} \cdot A,
$$

n'est en particulier pas bornée. Le vide quantique décrit par le modèle d'Euler-Heisenberg est donc instable.

En pratique, le calcul de l'énergie fondamentale du vide quantique devrait s'appuyer sur la théorie la plus précise pour décrire ce vide, à savoir l'électrodynamique quantique. 
Schwinger [31] a recouru à cette théorie pour obtenir par la méthode dite du temps propre une nouvelle dérivation de l'énergie d'Euler-Heisenberg, toujours dans la limite de champs électromagnétiques faiblement variables.

Notre objectif principal est de présenter une dérivation rigoureuse de l'énergie d'EulerHeisenberg dans le cas de champs purement magnétiques, stationnaires et, bien sûr, faiblement variables. Cette dérivation s'appuie sur l'introduction du modèle de Pauli-Villars [17] (cf. aussi $[16,25]$ ). À partir de l'électrodynamique quantique, ce modèle fournit une description rigoureuse du vide quantique en présence de champs électromagnétiques classiques et stationnaires. Avant d'exposer nos principaux résultats quant à la dérivation de l'énergie d'Euler-Heisenberg, nous allons commencer par rappeler les principaux aspects du modèle de Pauli-Villars.

\section{Le modèle de Pauli-Villars}

\subsection{La dérivation du modèle de Pauli-Villars}

Commençons par la détermination de l'énergie du vide quantique en présence d'un potentiel électromagnétique $\boldsymbol{A}$ classique et stationnaire. Dans le formalisme de la seconde quantification de la théorique quantique des champs, le vide quantique est décrit par le Hamiltonien

$$
\mathbb{H}_{\boldsymbol{A}}:=\frac{1}{2} \int_{\mathbb{R}^{3}}\left(\left\langle\Psi^{*}(x), D_{m, e \boldsymbol{A}} \Psi(x)\right\rangle_{\mathbb{C}^{4}}-\left\langle\Psi(x), D_{m, e \boldsymbol{A}} \Psi^{*}(x)\right\rangle_{\mathbb{C}^{4}}\right) d x .
$$

Ce Hamiltonien agit sur l'espace de Fock fermionique

$$
\mathcal{F}:=\bigoplus_{N=0}^{+\infty} \bigwedge_{1}^{N} L^{2}\left(\mathbb{R}^{3}, \mathbb{C}^{4}\right) .
$$

Les notations $\Psi(x)$ et $\Psi^{*}(x)$ qui apparaissent dans sa définition désignent les opérateurs de champ qui détruisent, respectivement créent, un électron à la position $x$. Le Hamiltonien $\mathbb{H}_{\boldsymbol{A}}$ prend en compte les interactions entre les électrons à travers le champ électromagnétique, mais ne tient pas compte d'interactions instantanées entre les électrons.

Dans la formule (3), l'opérateur de Dirac électromagnétique $D_{m, e \boldsymbol{A}}$ est défini par

$$
D_{m, e \boldsymbol{A}}:=D_{m, 0}-e \boldsymbol{\alpha} \cdot A+e V .
$$

L'opérateur de Dirac libre est ici donné par la formule

$$
D_{m, 0}=\boldsymbol{\alpha} \cdot(-i \nabla)+m \boldsymbol{\beta},
$$

dans laquelle $m$ désigne la masse d'un électron. La constante réduite de Planck $\hbar$ et la vitesse de la lumière $c$ sont omises par un choix convenable d'unités physiques. Les quatre matrices de Dirac $\boldsymbol{\alpha}_{1}, \boldsymbol{\alpha}_{2}, \boldsymbol{\alpha}_{3}$ et $\boldsymbol{\beta}$ sont égales à

$$
\boldsymbol{\alpha}_{k}=\left(\begin{array}{cc}
0 & \boldsymbol{\sigma}_{k} \\
\boldsymbol{\sigma}_{k} & 0
\end{array}\right) \text { et } \boldsymbol{\beta}=\left(\begin{array}{cc}
I_{2} & 0 \\
0 & -I_{2}
\end{array}\right),
$$

où les matrices de Pauli $\sigma_{1}, \sigma_{2}$ et $\sigma_{3}$ sont définies par

$$
\boldsymbol{\sigma}_{1}=\left(\begin{array}{ll}
0 & 1 \\
1 & 0
\end{array}\right), \boldsymbol{\sigma}_{2}=\left(\begin{array}{cc}
0 & -i \\
i & 0
\end{array}\right) \text { et } \boldsymbol{\sigma}_{3}=\left(\begin{array}{cc}
1 & 0 \\
0 & -1
\end{array}\right) \text {. }
$$


L'opérateur $D_{m, 0}$ est auto-adjoint sur l'espace des spineurs $L^{2}\left(\mathbb{R}^{3}, \mathbb{C}^{4}\right)$. En l'absence d'interaction, il donne la valeur de l'énergie d'un électron caractérisé par le spineur $\psi$ suivant la formule

$$
E(\psi):=\left\langle D_{m, 0}(\psi), \psi\right\rangle_{L^{2}} .
$$

Le spectre de l'opérateur $D_{m, 0}$ fournit ainsi les niveaux d'énergie possibles. Ce spectre est égal à

$$
\left.\left.\sigma\left(D_{m, 0}\right)=\right]-\infty,-m\right] \cup[m,+\infty[,
$$

et n'est donc pas minoré.

Dirac $[8,10,9]$ a postulé que les niveaux d'énergie négatifs sont occupés par une infinité d'électrons virtuels, de sorte qu'un électron "physique" a toujours une énergie positive. C'est l'image de la mer de Dirac dans laquelle le vide ne s'identifie plus à une quantité identiquement nulle, mais contient une infinité d'électrons virtuels. La polarisation du vide s'interprète alors comme une interaction entre ces électrons virtuels et le champ électromagnétique. Bien que la théorie quantique des champs ait reformulé cette interprétation, elle reste pertinente dans le cas du modèle de Pauli-Villars que nous allons maintenant continuer à introduire.

La différence qui apparaît dans la formule (3) résulte de la nécessité d'imposer l'invariance par conjugaison de la charge. Cette invariance exprime le fait que les lois de l'électromagnétisme sont inchangées lorsque les signes des charges et des champs électromagnétiques sont renversés. Elle se réduit ici à la formule

$$
\mathcal{C} \mathbb{H}_{\boldsymbol{A}} \mathcal{C}^{-1}=\mathbb{H}_{-\boldsymbol{A}},
$$

dans laquelle l'opérateur $\mathcal{C}$ désigne l'extension à l'espace de Fock $\mathcal{F}$ de l'opérateur de conjugaison de la charge défini sur l'espace des spineurs $L^{2}\left(\mathbb{R}^{3}, \mathbb{C}^{4}\right)$ par la formule

$$
\mathcal{C}(\psi):=i \boldsymbol{\beta} \boldsymbol{\alpha}_{2} \bar{\psi} .
$$

Le Hamiltonien $\mathbb{H}_{\boldsymbol{A}}$, qui dépend de manière quadratique des opérateurs de création $\Psi^{*}(x)$ et d'annihilation $\Psi(x)$, est bien compris [21]. La valeur moyenne de chaque état de l'espace de Fock $\mathcal{F}$ est donnée par l'expression formelle

$$
\left\langle\mathbb{H}_{\boldsymbol{A}}\right\rangle=\operatorname{tr} D_{m, e \boldsymbol{A}}\left(\gamma-\frac{1}{2}\right),
$$

dans laquelle la notation $\operatorname{tr} T$ renvoie à la trace de l'opérateur $T$. L'opérateur de densité à un corps $\gamma$ de l'état considéré vaut

$$
\gamma(x, y)_{i, j}=\left\langle\Psi_{i}^{*}(x) \Psi_{j}(y)\right\rangle,
$$

pour tout $1 \leq i, j \leq 4$ et tout $(x, y) \in \mathbb{R}^{6}$. Cet opérateur agit sur l'espace des spineurs $L^{2}\left(\mathbb{R}^{3}, \mathbb{C}^{4}\right)$. En raison du principe d'exclusion de Pauli, il satisfait la contrainte

$$
0 \leq \gamma \leq 1
$$

À chaque opérateur de densité à un corps $\gamma$ qui satisfait cette condition correspond un unique état de type Hartree-Fock généralisé [1]. Une approximation de ce type se révèle donc exacte ici, soit en l'absence de quantification du champ électromagnétique et des interactions instantanées entre les électrons. Dans la suite, nous abandonnons donc le formalisme de l'espace de Fock $\mathcal{F}$ pour nous contenter de celui des opérateurs de densité à un corps $\gamma$, dont dépend uniquement l'énergie. 
Le calcul de l'énergie du vide quantique se réduit alors à minimiser la valeur moyenne de la formule (4) par rapport à l'opérateur de densité $\gamma$. Ce calcul est explicite. Un minimiseur est donné par le projecteur spectral négatif

$$
\gamma_{\mathrm{vac}}=\mathbb{1}_{]-\infty, 0]}\left(D_{m, e \boldsymbol{A}}\right) .
$$

Nous retrouvons l'image de la mer de Dirac. En présence du potentiel électromagnétique $\boldsymbol{A}$, le vide quantique s'assimile à une infinité d'électrons virtuels qui remplissent complètement les niveaux d'énergie négatifs de l'opérateur de Dirac électromagnétique $D_{m, e \boldsymbol{A}}$.

L'énergie minimisante vaut

$$
\mathcal{F}_{\text {vac }}(e \boldsymbol{A})=-\frac{1}{2} \operatorname{tr}\left|D_{m, e \boldsymbol{A}}\right|,
$$

où la notation $|T|$ désigne la valeur absolue de l'opérateur $T$, soit la quantité $|T|:=\sqrt{T^{*} T}$. Cette énergie est infinie. Afin de lui donner un sens rigoureux, il est permis de redéfinir le niveau de référence du calcul des énergies. La référence la plus naturelle est donnée par le vide libre en l'absence de tout potentiel électromagnétique. L'énergie relative que nous obtenons alors

$$
\mathcal{F}_{\text {rel }}(e \boldsymbol{A}):=\frac{1}{2} \operatorname{tr}\left(\left|D_{m, 0}\right|-\left|D_{m, e \boldsymbol{A}}\right|\right),
$$

demeure en général infinie en raison de divergences ultraviolettes.

Une façon élémentaire d'appréhender ces divergences consiste à développer la transformée de Fourier de l'opérateur $\left|D_{m, 0}\right|$ lorsque la variable de Fourier $p$ tend vers l'infini. Dans cette limite, cette transformée de Fourier diverge en effet suivant le développement asymptotique

$$
\widehat{\left|D_{m, 0}\right|}(p)=\sqrt{p^{2}+m^{2}}=|p|+\frac{m^{2}}{2|p|}+\underset{|p| \rightarrow+\infty}{\mathcal{O}}\left(\frac{1}{|p|^{3}}\right) .
$$

Afin de supprimer ces divergences, il s'agit d'introduire une coupure ultraviolette qui aura pour fonction d'éliminer les premiers termes de ce développement, et par suite de donner un sens rigoureux à l'énergie relative de la formule (5).

Nous recourons à une régularisation classique en électrodynamique quantique : la méthode de Pauli et Villars [29]. Revenons à la formule (6) et calculons une combinaison linéaire de cette formule pour trois masses distinctes $\left(m_{j}\right)_{0 \leq j \leq 2}$ et des coefficients $\left(c_{j}\right)_{0 \leq j \leq 2}$. Nous obtenons

$$
\sum_{j=0}^{2} c_{j}\left|\widehat{D_{m_{j}, 0}}\right|(p)=\left(\sum_{j=0}^{2} c_{j}\right)|p|+\frac{1}{2|p|}\left(\sum_{j=0}^{2} c_{j} m_{j}^{2}\right)+\underset{|p| \rightarrow+\infty}{\mathcal{O}}\left(\frac{1}{|p|^{3}}\right),
$$

de sorte que sous les conditions dites de Pauli-Villars,

$$
\sum_{j=0}^{2} c_{j}=\sum_{j=0}^{2} c_{j} m_{j}^{2}=0
$$

toute divergence disparaît. Nous gagnons même une intégrabilité de l'ordre de $1 /|p|^{3}$ lorsque $|p| \rightarrow+\infty$. Quitte à introduire plus de masses $m_{j}$ et de coefficients $c_{j}$, nous pourrions obtenir une intégrabilité encore plus importante, mais trois masses suffisent en pratique.

L'énergie de Pauli-Villars que nous allons analyser dans la suite prend donc la forme

$$
\mathcal{F}_{\text {vac }}^{\mathrm{PV}}(e \boldsymbol{A}):=\frac{1}{2} \operatorname{tr} \sum_{j=0}^{2} c_{j}\left(\left|D_{m_{j}, 0}\right|-\left|D_{m_{j}, e \boldsymbol{A}}\right|\right) .
$$


L'indice 0 correspond aux termes "physiques" qui apparaissent dans l'énergie relative (5). Le coefficient $c_{0}$ est ainsi égal à 1 , tandis que la masse $m_{0}$ est celle de l'électron $m$. Les autres indices s'interprètent comme l'introduction de deux particules auxiliaires de masses $m_{1}$ et $m_{2}$. Ces particules n'ont pas de signification physique, mais permettent un calcul rigoureux de l'énergie du vide quantique. Les masses $m_{1}$ et $m_{2}$ sont choisies très grandes. Un tel ajout de particules très massives modifie peu les propriétés physiques du modèle dans le régime usuel des faibles énergies.

Nous avons pris la liberté de nommer ici, modèle de Pauli-Villars, ce modèle pour la description du vide quantique, puisqu'il repose sur la régularisation du même nom. L'électrodynamique quantique emploie diverses techniques de régularisation, parmi lesquelles la régularisation dimensionnelle [20] et la régularisation sur réseau [34]. La méthode de Pauli-Villars présente l'avantage d'une relative simplicité mathématique. Elle conserve de plus l'invariance par conjugaison de la charge, ainsi que l'invariance de jauge. La valeur de l'énergie n'est pas modifiée lorsque le potentiel magnétique $A$ est remplacé par un potentiel de la forme $A+\nabla f$. Cette invariance joue un rôle fondamental dans l'analyse de la fonctionnelle $\mathcal{F}_{\text {vac }}^{\mathrm{PV}}$, que nous allons désormais exposer.

\subsection{L'analyse mathématique du modèle de Pauli-Villars}

Le cadre fonctionnel pour l'analyse du modèle de Pauli-Villars se déduit de la forme de l'action lagrangienne classique $\mathcal{L}_{\text {clas }}$. Afin que cette dernière soit bien définie, il est nécessaire que le champ électromagnétique $\boldsymbol{F}$ soit de carré intégrable. Dans la jauge de Coulomb, ceci revient à supposer que le potentiel électrique $V$ est dans l'espace $\dot{H}^{1}\left(\mathbb{R}^{3}\right)$, tandis que le potentiel magnétique $A$ appartient à l'espace $\dot{H}_{\text {div }}^{1}\left(\mathbb{R}^{3}\right)$. Dans ce cadre, nous avons établi que l'énergie de Pauli-Villars $\mathcal{F}_{\text {vac }}^{\mathrm{PV}}$ est bien définie et satisfait les propriétés suivantes.

Théorème 1 ([17]). Supposons que les coefficients $\left(c_{j}\right)_{0 \leq j \leq 2}$ et les masses $\left(m_{j}\right)_{0 \leq j \leq 2}$ satisfont les conditions

$$
c_{0}=1, \quad m_{0}=m<m_{1}<m_{2}, \quad \text { et } \quad \sum_{j=0}^{2} c_{j}=\sum_{j=0}^{2} c_{j} m_{j}^{2}=0 .
$$

(i) Étant donné un potentiel électromagnétique $\boldsymbol{A} \in L^{1}\left(\mathbb{R}^{3}\right)^{4} \cap \dot{H}^{1}\left(\mathbb{R}^{3}\right) \times \dot{H}_{\text {div }}^{1}\left(\mathbb{R}^{3}\right)$, considérons l'opérateur

$$
T_{e \boldsymbol{A}}:=\frac{1}{2} \sum_{j=0}^{2} c_{j}\left(\left|D_{m_{j}, 0}\right|-\left|D_{m_{j}, e \boldsymbol{A}}\right|\right) .
$$

L'opérateur $\operatorname{tr}_{\mathbb{C}^{4}} T_{e \boldsymbol{A}}$ est à trace sur l'espace $L^{2}\left(\mathbb{R}^{3}, \mathbb{C}\right)$. En particulier, l'énergie de PauliVillars $\mathcal{F}_{\mathrm{vac}}^{\mathrm{PV}}(\boldsymbol{e} \boldsymbol{A})$ est bien définie par la formule

$$
\mathcal{F}_{\text {vac }}^{\mathrm{PV}}(e \boldsymbol{A}):=\operatorname{tr}\left(\operatorname{tr}_{\mathbb{C}^{4}} T_{e \boldsymbol{A}}\right) .
$$

(ii) L'énergie de Pauli-Villars $\mathcal{F}_{\mathrm{vac}}^{\mathrm{PV}}$ s'étend de manière unique en une application continue sur l'espace $\dot{H}^{1}\left(\mathbb{R}^{3}\right) \times \dot{H}_{\text {div }}^{1}\left(\mathbb{R}^{3}\right)$, qui se décompose sous la forme

$$
\mathcal{F}_{\text {vac }}^{\mathrm{PV}}(e \boldsymbol{A})=\mathcal{F}_{2}(e \boldsymbol{F})+\mathcal{R}(e \boldsymbol{A}) .
$$

(iii) Il existe une constante universelle $K$ telle que le reste $\mathcal{R}$ satisfait

$$
|\mathcal{R}(e \boldsymbol{A})| \leq K\left(\left(\sum_{j=0}^{2} \frac{\left|c_{j}\right|}{m_{j}}\right)\|e \boldsymbol{F}\|_{L^{2}}^{4}+\left(\sum_{j=0}^{2} \frac{\left|c_{j}\right|}{m_{j}^{2}}\right)\|e \boldsymbol{F}\|_{L^{2}}^{6}\right) .
$$


(iv) La fonctionnelle $\mathcal{F}_{2}$ est donnée par la formule

$$
\mathcal{F}_{2}(e \boldsymbol{F})=\frac{e^{2}}{8 \pi} \int_{\mathbb{R}^{3}} M(k)\left(|\widehat{B}(k)|^{2}-|\widehat{E}(k)|^{2}\right) d k,
$$

dans laquelle le multiplicateur de Fourier $M$ vaut

$$
M(k)=-\frac{2}{\pi} \sum_{j=0}^{2} c_{j} \int_{0}^{1} u(1-u) \ln \left(m_{j}^{2}+u(1-u)|k|^{2}\right) d u .
$$

Remarque. Soulignons l'introduction de la trace matricielle $\operatorname{tr}_{\mathbb{C}^{4}}$ dans la formule (10). Il est probable que l'opérateur $T_{e} \boldsymbol{A}$ ne soit pas à trace sans prendre d'abord cette trace matricielle. Dans le cas où il est à trace, cette introduction ne modifie en rien la valeur de l'énergie de Pauli-Villars $\mathcal{F}_{\text {vac }}^{\mathrm{PV}}$. Ce cas se produit lorsque le potentiel électrique $V$ est identiquement nul. L'opérateur $T_{e \boldsymbol{A}}$ est alors à trace sur $L^{2}\left(\mathbb{R}^{3}, \mathbb{C}\right)$, et l'énergie de PauliVillars est donnée par les formules (8) ou (10).

La preuve du théorème 1 repose sur un développement à l'ordre six de l'opérateur $T_{e} \boldsymbol{A}$ par rapport aux puissances du potentiel électromagnétique $\boldsymbol{A}$. Lorsque ce potentiel est intégrable, la trace $\operatorname{tr}_{\mathbb{C}^{4}}$ de chacun des termes de ce développement est un opérateur à trace sur $L^{2}\left(\mathbb{R}^{3}, \mathbb{C}\right)$. La trace des termes impairs est nulle de par l'invariance par conjugaison de la charge, tandis que l'invariance de jauge permet d'établir que le terme du second ordre prend la forme (12). Il ne reste ensuite qu'à estimer les termes d'ordre quatre et six suivant l'inégalité (11) pour conclure la preuve du théorème 1. Nous renvoyons à [17] pour de plus amples détails.

Le multiplicateur de Fourier $M$ qui apparaît dans le terme du second ordre $\mathcal{F}_{2}$, décrit la réponse linéaire du vide quantique au champ électromagnétique $\boldsymbol{F}$. Ce multiplicateur satisfait la borne uniforme

$$
0<M(k) \leq M(0)=\frac{2 \ln (\Lambda)}{3 \pi} .
$$

La coupure ultraviolette moyenne $\Lambda$ est ici définie par la formule

$$
\ln (\Lambda):=-\sum_{j=0}^{2} c_{j} \ln \left(m_{j}\right)
$$

Dans la limite où cette coupure tend vers $+\infty$, soit dans la limite où les masses $m_{1}$ et $m_{2}$ sont très grandes, le multiplicateur $M$ vérifie

$$
\lim _{\Lambda \rightarrow \infty}\left(\frac{2 \ln \Lambda}{3 \pi}-M(k)\right)=U(k):=\frac{|k|^{2}}{4 \pi} \int_{0}^{1} \frac{z^{2}-z^{4} / 3}{1+|k|^{2}\left(1-z^{2}\right) / 4} d z .
$$

Le multiplicateur limite $U$ induit le célèbre potentiel de Uehling [32], qui est la contribution du premier ordre à la polarisation du vide.

Afin de décrire plus en détail ce phénomène, il convient d'introduire l'action lagrangienne effective associée à l'énergie de Pauli-Villars. Rappelons qu'en présence de densités externes de charge $\rho_{\text {ext }}$ et de courant $j_{\text {ext }}$, cette fonctionnelle est donnée par l'expression

$$
\mathcal{L}_{\text {eff }}^{\mathrm{PV}}(\boldsymbol{A})=-\mathcal{F}_{\text {vac }}^{\mathrm{PV}}(e \boldsymbol{A})+\frac{1}{8 \pi} \int_{\mathbb{R}^{3}}\left(|E|^{2}-|B|^{2}\right)-e \int_{\mathbb{R}^{3}}\left(\rho_{\text {ext }} V-j_{\text {ext }} \cdot A\right) .
$$


Elle est bien définie lorsque le potentiel électromagnétique $\boldsymbol{A}$ appartient à $\dot{H}^{1}\left(\mathbb{R}^{3}\right) \times$ $\dot{H}_{\text {div }}^{1}\left(\mathbb{R}^{3}\right)$ par le théorème 1 , et la fonction $\rho_{\text {ext }}$ et chacune des composantes de $j_{\text {ext }}$ sont dans l'espace dual de $\dot{H}^{1}\left(\mathbb{R}^{3}\right)$, soit dans l'espace de Coulomb

$$
\mathcal{C}\left(\mathbb{R}^{3}\right):=\left\{f \in \mathcal{S}^{\prime}\left(\mathbb{R}^{3}, \mathbb{R}\right), \text { t.q. }\|f\|_{\mathcal{C}}^{2}:=\int_{\mathbb{R}^{3}} \frac{|\widehat{f}(k)|^{2}}{|k|^{2}} d k<+\infty\right\} .
$$

La valeur du potentiel électromagnétique en interaction avec le vide quantique est donnée par un point critique $\boldsymbol{A}_{*}$ de cette fonctionnelle. D'après la dérivation précédente de l'énergie de Pauli-Villars, l'opérateur de densité à un corps $\gamma_{\text {vac }}$ du vide quantique est alors égal au projecteur spectral négatif

$$
\gamma_{\mathrm{vac}}=\mathbb{1}_{]-\infty, 0]}\left(D_{m, e \boldsymbol{A}}\right) .
$$

Pour des potentiels électromagnétiques $\boldsymbol{A}$ suffisamment faibles, l'action lagrangienne effective $\mathcal{L}_{\text {eff }}^{\mathrm{PV}}$ est une perturbation de l'action lagrangienne classique $\mathcal{L}_{\text {clas }}$. La façon la plus naturelle de construire un point critique $\boldsymbol{A}_{*}$ consiste donc à résoudre le problème de min-max

$$
\min _{A \in \dot{H}_{\mathrm{div}}^{1}\left(\mathbb{R}^{3}\right)} \max _{V \in \dot{H}^{1}\left(\mathbb{R}^{3}\right)} \mathcal{L}_{\mathrm{eff}}^{\mathrm{PV}}(\boldsymbol{A}) .
$$

Ceci conduit à l'énoncé suivant.

Théorème 2 ([17]). Supposons que les coefficients $\left(c_{j}\right)_{0 \leq j \leq 2}$ et les masses $\left(m_{j}\right)_{0 \leq j \leq 2}$ satisfont les conditions (9).

(i) Il existe un nombre strictement positif $r$ tel que, quelles que soient les densités de charge $\rho_{\text {ext }} \in \mathcal{C}\left(\mathbb{R}^{3}\right)$ et de courant $j_{\text {ext }} \in \mathcal{C}\left(\mathbb{R}^{3}\right)^{3}$ qui satisfont

$$
\left\|\rho_{\text {ext }}\right\|_{\mathcal{C}}^{2}+\left\|j_{\text {ext }}\right\|_{\mathcal{C}^{3}}^{2}<\frac{r^{2} m_{0}}{6^{4} \pi^{2} e^{4}}
$$

il existe une unique solution $\boldsymbol{A}_{*} \in \dot{H}^{1}\left(\mathbb{R}^{3}\right) \times \dot{H}_{\text {div }}^{1}\left(\mathbb{R}^{3}\right)$ au problème de min-max

$$
\begin{aligned}
& \mathcal{L}_{\text {eff }}^{\mathrm{PV}}\left(\boldsymbol{A}_{*}\right)=\max _{\|\nabla V\|_{L^{2}}<\frac{r \sqrt{m_{0}}}{3 e}} \inf _{\|\operatorname{rot} A\|_{L^{2}}<\frac{r \sqrt{m_{0}}}{3 e}} \mathcal{L}_{\text {eff }}^{\mathrm{PV}}(\boldsymbol{A}) \\
& =\min _{\|\operatorname{rot} A\|_{L^{2}}<\frac{r \sqrt{m_{0}}}{3 e}} \sup _{\|\nabla V\|_{L^{2}}<\frac{r \sqrt{m_{0}}}{3 e}} \mathcal{L}_{\text {eff }}^{\mathrm{PV}}(\boldsymbol{A}) .
\end{aligned}
$$

(ii) Le potentiel électromagnétique $\boldsymbol{A}_{*}$ est solution des équations de Poisson non linéaires

$$
\left\{\begin{array}{l}
-\Delta V_{*}=4 \pi e\left(\rho_{\mathrm{vac}}\left(e \boldsymbol{A}_{*}\right)+\rho_{\mathrm{ext}}\right), \\
-\Delta A_{*}=4 \pi e\left(j_{\mathrm{vac}}\left(e \boldsymbol{A}_{*}\right)+j_{\mathrm{ext}}\right) .
\end{array}\right.
$$

Dans ces équations, les densités de charge $\rho_{\mathrm{vac}}\left(e \boldsymbol{A}_{*}\right)$ et de courant $j_{\mathrm{vac}}\left(e \boldsymbol{A}_{*}\right)$ du vide quantique sont définies par les formules

$$
\rho_{\mathrm{vac}}\left(e \boldsymbol{A}_{*}\right)(x)=\operatorname{tr}_{\mathbb{C}^{4}}\left(\gamma_{e \boldsymbol{A}_{*}}(x, x)\right), \quad \text { et } \quad j_{\mathrm{vac}}\left(e \boldsymbol{A}_{*}\right)(x)=\operatorname{tr}_{\mathbb{C}^{4}}\left(\boldsymbol{\alpha} \gamma_{e \boldsymbol{A}_{*}}(x, x)\right),
$$

et elles appartiennent à l'espace de Coulomb $\mathcal{C}\left(\mathbb{R}^{3}\right)$. La fonction $\gamma_{e} \boldsymbol{A}_{*}$ qui apparaît ici, est le noyau intégral de l'opérateur

$$
\gamma_{e \boldsymbol{A}_{*}}=\sum_{j=0}^{2} c_{j} \mathbb{1}_{]-\infty, 0]}\left(D_{m_{j}, e \boldsymbol{A}_{*}}\right) .
$$

(iii) L'opérateur de densité à un corps $\gamma_{\mathrm{vac}}^{\mathrm{PV}}$ du vide quantique est égal au projecteur spectral négatif

$$
\gamma_{\mathrm{vac}}^{\mathrm{PV}}=\mathbb{1}_{]-\infty, 0]}\left(D_{m, e \boldsymbol{A}}\right)
$$


La preuve du théorème 2 repose sur des arguments classiques d'analyse convexe. L'action lagrangienne effective $\mathcal{L}_{\text {eff }}^{\mathrm{PV}}(\boldsymbol{A})$ garde en effet la géométrie de point selle de l'action lagrangienne classique $\mathcal{L}_{\text {clas }}(\boldsymbol{A})$ lorsque le potentiel électromagnétique $\boldsymbol{A}$ et les densités externes de charge $\rho_{\text {ext }}$ et de courant $j_{\text {ext }}$ sont petites. Cette affirmation résulte de la forme du terme du second ordre $\mathcal{F}_{2}(e \boldsymbol{F})$ donné par la formule (12), et de la positivité du multiplicateur de Fourier $M$. L'hypothèse de petitesse (13) permet de se placer dans le cas de potentiels électromagnétiques suffisamment petits pour que les techniques usuelles de l'analyse convexe fournissent une unique solution locale du problème de min-max (14). La résolution globale de ce problème de min-max, ainsi que sa résolution pour des densités externes de charge et de courant quelconques, restent à notre connaissance, ouvertes.

Pour conclure avec le modèle de Pauli-Villars, notons que, lorsque les densités externes de charge et de courant sont identiquement nulles, l'unique minimiseur $\boldsymbol{A}^{*}$ est lui-aussi identiquement nul. Le vide libre est donc représenté par le projecteur spectral négatif de l'opérateur de Dirac libre

$$
\gamma_{\text {vac }}^{0}=\mathbb{1}_{]-\infty, 0]}\left(D_{m, 0}\right),
$$

ce qui est cohérent avec l'image de la mer de Dirac.

\section{Le modèle d'Euler-Heisenberg (purement magnétique)}

\subsection{La dérivation du modèle d'Euler-Heisenberg à partir de celui de Pauli-Villars}

À partir de l'électrodynamique quantique, nous venons d'obtenir le modèle de PauliVillars qui décrit la polarisation du vide quantique en présence d'un champ électromagnétique classique. Ce modèle est défini rigoureusement sur le plan mathématique. Son étude aboutit à la construction d'un vide quantique en accord avec l'image de la mer de Dirac.

Toujours à partir de l'électrodynamique quantique, Schwinger [31] a dérivé le modèle d'Euler-Heisenberg dans un régime de champs électromagnétiques faiblement variables. Il est naturel de se demander si cette dérivation demeure possible à partir du modèle de Pauli-Villars. Une réponse positive à cette question fournit une dérivation rigoureuse du modèle d'Euler-Heisenberg. Elle donne aussi une justification de plus à l'introduction du modèle de Pauli-Villars en tant qu'approximation de l'électrodynamique quantique.

Afin de parvenir à une telle dérivation, nous nous restreignons au cas de champs purement magnétiques pour lesquels l'énergie d'Euler-Heisenberg $\mathcal{F}_{\text {vac }}^{\mathrm{EH}}$ est bien définie. Nous introduisons ensuite un petit paramètre $\varepsilon$ qui vise à quantifier la petitesse des variations du champ magnétique. Une des façons parmi les plus simples d'imposer cette petitesse consiste à considérer des potentiels électromagnétiques qui satisfont au changement d'échelles

$$
\boldsymbol{A}_{\varepsilon}(x):=\left(0, \frac{1}{\varepsilon} A(\varepsilon x)\right) .
$$

Le champ magnétique vaut alors

$$
B_{\varepsilon}(x):=[\operatorname{rot} A](\varepsilon x),
$$

de sorte que son gradient est de l'ordre de $\varepsilon$, lorsque les dérivées secondes du potentiel magnétique $A$ sont contrôlées.

Notre objectif est désormais d'étudier la limite de la quantité $\mathcal{F}_{\mathrm{vac}}^{\mathrm{PV}}\left(e \boldsymbol{A}_{\varepsilon}\right)$ lorsque le paramètre $\varepsilon$ tend vers 0 , et de vérifier que ce régime est gouverné par l'énergie d'EulerHeisenberg. 
Le changement de variable $x_{\varepsilon}:=\varepsilon x$ permet de mieux comprendre la nature de ce régime. La fonctionnelle $\mathcal{F}_{\text {vac }}^{\mathrm{PV}}\left(e \boldsymbol{A}_{\varepsilon}\right)$ s'écrit alors sous la forme

$$
\mathcal{F}_{\mathrm{vac}}^{\mathrm{PV}}\left(e \boldsymbol{A}_{\varepsilon}\right)=\frac{1}{2} \operatorname{tr} \sum_{j=0}^{2} c_{j}\left(\left|D_{m_{j}, 0}^{\varepsilon}\right|-\left|D_{m_{j}, e A}^{\varepsilon}\right|\right) .
$$

Dans cette expression, l'opérateur de Dirac magnétique $D_{m, e A}^{\varepsilon}$ est défini par la formule

$$
D_{m, e A}^{\varepsilon}:=\boldsymbol{\alpha} \cdot\left(-i \varepsilon \nabla-\frac{e}{\varepsilon} A\right)+m \boldsymbol{\beta} .
$$

Nous sommes donc amenés à étudier un régime semi-classique lorsque le petit paramètre $\varepsilon$ tend vers 0 . Nous observons de plus que le paramètre $\varepsilon$ apparaît au dénominateur du potentiel magnétique, de sorte que nous avons aussi affaire à un régime de champs magnétiques forts.

Yngvason [35], Lieb, Solovej et Yngvason [26], puis Erdös et Solovej [11, 12, 13], ont analysé en détail les propriétés spectrales des opérateurs de Pauli électromagnétiques dans ce régime semi-classique avec champ magnétique fort. Ces travaux reposent en particulier sur l'utilisation d'opérateurs cohérents magnétiques, sur lesquels nous reviendrons dans la suite. L'extension de ces techniques à l'opérateur de Dirac magnétique $D_{m, e A}^{\varepsilon}$ permet une dérivation rigoureuse d'un modèle de type Euler-Heisenberg dans la limite où le petit paramètre $\varepsilon$ tend vers 0 .

Théorème 3 ([18]). Soit $B=\operatorname{rot} A \in \mathcal{C}^{0}\left(\mathbb{R}^{3}\right)^{3}$, avec $\operatorname{div} A=0$, tel que

$$
B \in L^{1}\left(\mathbb{R}^{3}\right)^{3} \cap L^{\infty}\left(\mathbb{R}^{3}\right)^{3} \quad \text { et } \quad \nabla B \in L^{1}\left(\mathbb{R}^{3}\right)^{9} \cap L^{6}\left(\mathbb{R}^{3}\right)^{9} .
$$

La version régularisée par la méthode de Pauli-Villars de l'énergie d'Euler-Heisenberg (purement magnétique)

$\mathcal{F}_{\text {vac }}^{\mathrm{rEH}}(e A)=\int_{\mathbb{R}^{3}} f_{\text {vac }}^{\mathrm{rEH}}(e B):=\frac{1}{8 \pi^{2}} \int_{\mathbb{R}^{3}} \int_{0}^{\infty} d s s^{-3}\left(\sum_{j=0}^{2} c_{j} e^{-s m_{j}^{2}}\right)(e s|B| \operatorname{coth}(e s|B|)-1)$,

est bien définie, et elle satisfait

$$
\varepsilon^{3} \mathcal{F}_{\text {vac }}^{\mathrm{PV}}\left(e \boldsymbol{A}_{\varepsilon}\right)=\mathcal{F}_{\text {vac }}^{\mathrm{rEH}}(e A)+\underset{\varepsilon \rightarrow 0}{\mathcal{O}}(\varepsilon) .
$$

Ce n'est donc pas la version initiale de l'énergie d'Euler-Heisenberg $\mathcal{F}_{\text {vac }}^{\mathrm{EH}}$ qui apparaît dans la limite $\varepsilon \rightarrow 0$, mais sa version régularisée $\mathcal{F}_{\text {vac }}^{\mathrm{rEH}}$ par la méthode de Pauli-Villars. Comme les coefficients $\left(c_{j}\right)_{0 \leq j \leq 2}$ et les masses $\left(m_{j}\right)_{0 \leq j \leq 2}$ introduits par cette régularisation ne dépendent pas du paramètre $\varepsilon$, il n'y a bien sûr aucune raison qu'ils disparaissent lorsque celui-ci tend vers 0 . Afin de compléter la dérivation du modèle initial d'Euler-Heisenberg, nous réaliserons une renormalisation multiplicative de la charge dans le paragraphe 3.2 comme il est d'usage en électrodynamique quantique.

Les conditions de Pauli-Villars (7) conduisent à l'inégalité

$$
\sum_{j=0}^{2} c_{j} e^{-s m_{j}^{2}} \geq 0
$$

pour tout nombre positif $s$. La densité d'énergie par unité de volume $f_{\text {vac }}^{\mathrm{rEH}}$ est par conséquent positive. Au contraire de l'action lagrangienne $\mathcal{L}_{\text {eff }}^{\mathrm{EH}}$, la version régularisée de l'action lagrangienne

$$
\mathcal{L}_{\text {eff }}^{\mathrm{rEH}}(A):=-\int_{\mathbb{R}^{3}} f_{\mathrm{vac}}^{\mathrm{rEH}}(e B)-\frac{1}{8 \pi} \int_{\mathbb{R}^{3}}|B|^{2}+e \int_{\mathbb{R}^{3}} j_{\text {ext }} \cdot A,
$$


est donc majorée lorsque le champ magnétique $B$ est de carré intégrable et que la densité externe de courant $j_{\text {ext }}$ appartient à l'espace de Coulomb $\mathcal{C}\left(\mathbb{R}^{3}\right)^{3}$. Le vide quantique associé à cette version régularisée du modèle d'Euler-Heisenberg est ainsi stable.

La densité d'énergie par unité de volume $f_{\text {vac }}^{\mathrm{rEH}}$ se comporte de plus comme

$$
f_{\text {vac }}^{\mathrm{rEH}}(e B) \sim-\frac{e^{2}}{12 \pi^{2}}\left(\sum_{j=0}^{2} c_{j} \ln \left(m_{j}\right)\right)|B|^{2},
$$

lorsque le champ magnétique $|B| \rightarrow 0$, et

$$
f_{\text {vac }}^{\mathrm{rEH}}(e B) \sim \frac{e}{4 \pi^{2}}\left(\sum_{j=0}^{2} c_{j} m_{j}^{2} \ln \left(m_{j}\right)\right)|B|,
$$

quand $|B| \rightarrow+\infty$. L'énergie régularisée $\mathcal{F}_{\text {vac }}^{\mathrm{rEH}}$ est donc bien définie sous la condition

$$
\int_{\mathbb{R}^{3}} \frac{|B|^{2}}{1+|B|}<+\infty
$$

Il est légitime de se demander si le résultat de convergence du théorème 3 demeure valable sous cette seule hypothèse.

Les hypothèses (15) sur le champ magnétique $B$ sont techniques, et certainement pas optimales. Les différents espaces de Lebesgue qui apparaissent dans ces hypothèses sont nécessaires pour contrôler les termes de reste qui apparaissent à la limite $\varepsilon \rightarrow 0$ par un facteur de l'ordre de $\mathcal{O}(\varepsilon)$.

Notons néanmoins que le caractère borné du champ magnétique est crucial afin de s'appuyer sur les méthodes d'opérateurs cohérents magnétiques qui sont à la base de la preuve du théorème 3 . Il est de plus raisonnable de contrôler le gradient du champ magnétique dans un régime où celui-ci est supposé faiblement variable.

Avant de revenir plus en détail sur la preuve du théorème 3 au paragraphe 3.3, il est temps de compléter la dérivation du modèle original d'Euler-Heisenberg, soit de dépasser l'obstacle de la régularisation de Pauli-Villars, nécessaire à la définition du modèle du même nom.

\subsection{La renormalisation du modèle d'Euler-Heisenberg}

Puisque la méthode de régularisation de Pauli-Villars consiste en l'ajout de particules très massives, il est légitime d'espérer s'en abstraire en prenant la limite où leurs masses $m_{1}$ et $m_{2}$ tendent vers $+\infty$. Cette possibilité s'avère illusoire en pratique. Lorsque $m_{1} \rightarrow+\infty$ et $m_{2} \rightarrow+\infty$, la quantité $\mathcal{F}_{\text {vac }}^{\mathrm{rEH}}(e A)$ se développe sous la forme

$$
\mathcal{F}_{\text {vac }}^{\mathrm{rEH}}(e A)=\frac{e^{2}}{12 \pi^{2}} \ln \Lambda \int_{\mathbb{R}^{3}}|B|^{2}+\mathcal{F}_{\text {vac }}^{\mathrm{EH}}(e A)+\underset{m_{1}, m_{2} \rightarrow+\infty}{o}(1) .
$$

L'énergie d'Euler-Heisenberg apparaît certes au second ordre de ce développement, mais le terme du premier ordre présente une divergence logarithmique par rapport à la coupure ultraviolette moyenne $\Lambda$.

Une telle divergence est aussi présente en électrodynamique quantique. Pour la contourner, cette théorie s'appuie sur une méthode dite de renormalisation multiplicative de la charge [19, Paragraphe 5.2]. Cette procédure que nous allons maintenant détailler conduit 
à un lien perturbatif entre les versions originales et régularisées de l'énergie d'EulerHeisenberg.

Commençons par développer l'action lagrangienne renormalisée dans la limite où $m_{1} \rightarrow$ $+\infty$ et $m_{2} \rightarrow+\infty$. Nous obtenons l'expression

$$
\mathcal{L}_{\text {vac }}^{\mathrm{rEH}}(A)=-\frac{1}{8 \pi}\left(1+\frac{2 e^{2}}{3 \pi} \ln \Lambda\right) \int_{\mathbb{R}^{3}}|B|^{2}+e \int_{\mathbb{R}^{3}} j_{\mathrm{ext}} \cdot A-\mathcal{F}_{\mathrm{vac}}^{\mathrm{EH}}(e A)+\underset{m_{1}, m_{2} \rightarrow+\infty}{O}(1) .
$$

En principe, l'énergie d'Euler-Heisenberg $\mathcal{F}_{\text {vac }}^{\mathrm{EH}}(e A)$ apporte des corrections non linéaires à l'action classique $\mathcal{L}_{\text {clas }}(A)$, ce qui n'est pas le cas ici.

Afin de remédier à cette difficulté, il suffit d'admettre que le potentiel $A$ et le champ $B$ qui apparaissent dans l'action lagrangienne $\mathcal{L}_{\text {vac }}^{\mathrm{rEH}}(A)$ ne sont pas ceux qui sont observés en pratique. Le potentiel magnétique "physique" $A_{\mathrm{ph}}$ et le champ magnétique $B_{\mathrm{ph}}$ qui lui est associé, doivent être tels que les deux premiers termes du développement (16) soient égaux à l'action classique $\mathcal{L}_{\text {clas }}\left(A_{\mathrm{ph}}\right)$. Cette affirmation conduit aux formules

$$
A_{\mathrm{ph}}:=\left(1+\frac{2 e^{2}}{3 \pi} \ln \Lambda\right)^{\frac{1}{2}} A, \quad \text { et } \quad B_{\mathrm{ph}}:=\left(1+\frac{2 e^{2}}{3 \pi} \ln \Lambda\right)^{\frac{1}{2}} B
$$

ainsi qu'à l'introduction d'une charge élémentaire "physique" $e_{\mathrm{ph}}$, laquelle est donnée par l'identité

$$
e_{\mathrm{ph}}^{2}:=\frac{e^{2}}{1+\frac{2 e^{2}}{3 \pi} \ln \Lambda}
$$

L'électrodynamique quantique justifie l'introduction de ces quantités "physiques" à l'aide de développement perturbatifs dans la limite $e_{\mathrm{ph}} \rightarrow 0$. Ces développements sont en excellente adéquation avec les observations expérimentales [30, Paragraphe 6.3].

Qu'en est-il pour le modèle d'Euler-Heisenberg? Pour répondre à cette question, nous devons déterminer un développement perturbatif de la quantité $\mathcal{L}_{\text {eff }}^{\mathrm{rEH}}(A)$ lorsque $e_{\mathrm{ph}} \rightarrow$ 0 . Afin de calculer ce développement, il reste à éliminer la dépendance en la coupure ultraviolette moyenne $\Lambda$. Nous avons vu précédemment qu'il est vain de passer à la limite $\Lambda \rightarrow+\infty$.

Lors d'une renormalisation multiplicative de la charge, l'élimination de cette coupure repose sur l'hypothèse que la charge élémentaire "physique" $e_{\mathrm{ph}}$ est égale à la charge élémentaire $e$ du modèle original à un changement d'unité près. Cette affirmation revient à l'introduction d'un nombre $\left.Z_{3} \in\right] 0,1[$ tel que

$$
e_{\mathrm{ph}}^{2}=Z_{3} e^{2} .
$$

À l'aide de la formule (17), nous pouvons vérifier que la coupure ultraviolette moyenne vaut alors

$$
\Lambda=e^{\frac{3 \pi\left(1-Z_{3}\right)}{2 e_{\mathrm{ph}}^{2}}} .
$$

La différence entre l'action lagrangienne renormalisée $\mathcal{L}_{\text {eff }}^{\mathrm{rEH}}(A)$ et l'action lagrangienne initiale (dans les variables physiques) $\mathcal{L}_{\text {eff }}^{\mathrm{EH}}\left(A_{\mathrm{ph}}\right)$ est contrôlée par des puissances négatives de la coupure $\Lambda$. Il résulte de la formule (18) que le développement de ces puissances dans la limite $e_{\mathrm{ph}} \rightarrow 0$ est identiquement nul lorsque le nombre $Z_{3}$ est fixé. Les quantités $\mathcal{L}_{\text {eff }}^{\mathrm{rEH}}(A)$ et $\mathcal{L}_{\text {eff }}^{\mathrm{EH}}\left(A_{\mathrm{ph}}\right)$ sont donc perturbativement égales.

Théorème 4 ([18]). Soit $j_{\mathrm{ext}} \in \mathcal{C}\left(\mathbb{R}^{3}\right)^{3}$ et $A \in \dot{H}_{\text {div }}^{1}\left(\mathbb{R}^{3}\right)$, avec $B:=\operatorname{rot} A \in L^{4}\left(\mathbb{R}^{3}\right)$. Il existe une constante universelle $C$ telle que

$$
\left|\mathcal{L}_{\text {eff }}^{\mathrm{rEH}}(A)-\mathcal{L}_{\mathrm{eff}}^{\mathrm{EH}}\left(A_{\mathrm{ph}}\right)\right| \leq C\left|c_{1}\right|\left(\frac{e_{\mathrm{ph}}}{m}\right)^{4} e^{-\frac{6 \pi\left(1-Z_{3}\right)}{e_{\mathrm{ph}}^{2}}} \int_{\mathbb{R}^{3}}\left|B_{\mathrm{ph}}\right|^{4}
$$


En conclusion, l'action d'Euler-Heisenberg $\mathcal{L}_{\text {eff }}^{\mathrm{EH}}\left(A_{\mathrm{ph}}\right)$ est perturbativement égale à la limite de l'action de Pauli-Villars dans le régime des champs magnétiques faiblement variables après renormalisation multiplicative de la charge. C'est par cette procédure que s'achève cette dérivation rigoureuse du modèle d'Euler-Heisenberg dans le cas purement magnétique.

Remarque. Pour chaque entier $d \geq 2$, le développement asymptotique de l'action lagrangienne $\mathcal{L}_{\text {eff }}^{\mathrm{EH}}\left(A_{\mathrm{ph}}\right)$ est donnée par la formule

$$
\mathcal{L}_{\text {eff }}^{\mathrm{EH}}\left(A_{\mathrm{ph}}\right)=\mathcal{L}_{\text {clas }}\left(A_{\mathrm{ph}}\right)-\frac{m^{4}}{8 \pi^{2}} \sum_{n=2}^{d} \frac{4^{n} \mathcal{B}_{2 n} e_{\mathrm{ph}}^{2 n}}{2 n(2 n-1)(2 n-2) m^{2 n}} \int_{\mathbb{R}^{3}}|B|^{2 n}+\underset{e_{\mathrm{ph}} \rightarrow 0}{o}\left(e_{\mathrm{ph}}^{2 d}\right),
$$

lorsque le nombre $Z_{3}$ reste fixé. Dans cette expression, les nombres de Bernoulli $\mathcal{B}_{2 n}$ sont égaux à

$$
\mathcal{B}_{2 n}=(-1)^{n+1} \frac{(2 n) !}{(2 \pi)^{2 n}} \zeta(2 n),
$$

où $\zeta$ désigne la fonction zêta de Riemann.

Les termes de ce développement ne dépendent pas du choix du nombre $Z_{3}$. Cette propriété est une conséquence de la formule (18). En d'autres termes, la valeur précise de la coupure ultraviolette moyenne $\Lambda$ importe peu à un niveau perturbatif, dès l'instant où elle correspond à une renormalisation multiplicative de la charge.

Notons enfin que la série associée au développement (19) n'est pas convergente. Elle est par contre sommable au sens de Borel [6], et sa somme de Borel est exactement l'action lagrangienne $\mathcal{L}_{\text {eff }}^{\mathrm{EH}}\left(A_{\mathrm{ph}}\right)$.

\subsection{Quelques éléments de la preuve du théorème 3}

La preuve du théorème 3 repose sur l'utilisation d'opérateurs cohérents magnétiques introduits dans les travaux de Lieb, Solovej et Yngvason [35, 26]. Ces opérateurs fournissent une approximation de l'identité qui induit une localisation dans l'espace direct et une projection sur les états propres d'un opérateur sous-jacent. Afin de clarifier la présentation, nous introduisons d'abord la procédure de localisation, avant de traiter de la projection sur les états propres de l'opérateur de Pauli magnétique qui nous intéresse ici.

Considérons un nombre strictement positif $\rho$. La fonction gaussienne définie par l'expression

$$
G_{\rho}(x):=(\pi \rho)^{-\frac{3}{2}} e^{-\frac{|x|^{2}}{\rho^{2}}}
$$

satisfait l'identité

$$
\int_{\mathbb{R}^{3}} G_{\rho}(x-y)^{2} d y=1
$$

pour tout vecteur $x \in \mathbb{R}^{3}$. Cette formule fournit une approximation intégrale de l'identité dans l'espace direct, qui permet une localisation autour de chaque vecteur $y \in \mathbb{R}^{3}$ sur une échelle de l'ordre de $\rho$. Le choix particulier de la fonction gaussienne $G_{\rho}$ résulte de la volonté de conserver l'invariance par rotation lors de cette localisation.

Cette approximation intégrale de l'identité rend possible une localisation de l'opérateur $T_{e \boldsymbol{A}}$ qui apparaît dans la définition (8) de l'énergie de Pauli-Villars $\mathcal{F}_{\text {vac }}^{\mathrm{PV}}(e \boldsymbol{A})$. Au moins formellement, cette quantité s'écrit en effet sous la forme

$$
\mathcal{F}_{\text {vac }}^{\mathrm{PV}}(e \boldsymbol{A})=\operatorname{tr} T_{e \boldsymbol{A}}=\int_{\mathbb{R}^{3}} \operatorname{tr}_{\mathbb{C}^{4}} T_{e \boldsymbol{A}}(x, x) d x,
$$


où la fonction $T_{e \boldsymbol{A}}\left(x, x^{\prime}\right)$ est le noyau intégral de l'opérateur $T_{e \boldsymbol{A}}$. L'introduction de l'identité (20) dans cette expression conduit à la formule

$$
\mathcal{F}_{\mathrm{vac}}^{\mathrm{PV}}(e \boldsymbol{A})=\int_{\mathbb{R}^{3}}\left(\int_{\mathbb{R}^{3}} \operatorname{tr}_{\mathbb{C}^{4}} G_{\rho}(x-y) T_{e \boldsymbol{A}}(x, x) G_{\rho}(x-y) d x\right) d y:=\int_{\mathbb{R}^{3}} f_{\mathrm{vac}}^{\mathrm{PV}}(e \boldsymbol{A})(y) d y,
$$

après application du théorème de Fubini. La densité d'énergie $f_{\text {vac }}^{\mathrm{PV}}(e \boldsymbol{A})$ s'identifie à la trace d'un opérateur localisé

$$
f_{\mathrm{vac}}^{\mathrm{PV}}(e \boldsymbol{A})(y)=\operatorname{tr} G_{\rho}(\cdot-y) T_{e \boldsymbol{A}} G_{\rho}(\cdot-y),
$$

pour chaque vecteur $y \in \mathbb{R}^{3}$. Cette formule se justifie rigoureusement lorsque l'opérateur $T_{e \boldsymbol{A}}$ est à trace. Elle ramène l'analyse de la fonctionnelle $\mathcal{F}_{\text {vac }}^{\mathrm{PV}}(e \boldsymbol{A})$ à l'étude de densités $f_{\text {vac }}^{\mathrm{PV}}(e \boldsymbol{A})(y)$ pour lesquelles les interactions électromagnétiques dans l'espace direct sont localisées autour de chaque vecteur $y$.

Dans le régime des faibles variations du champ magnétique où le potentiel électromagnétique s'écrit sous la forme

$$
\boldsymbol{A}_{\varepsilon}(x)=\left(0, \frac{1}{\varepsilon} A(\varepsilon x)\right)
$$

nous arrivons à la formule

$$
\varepsilon^{3} f_{\mathrm{vac}}^{\mathrm{PV}}\left(e \boldsymbol{A}_{\varepsilon}\right)(y)=\frac{1}{2} \operatorname{tr} \sum_{j=0}^{2} c_{j} G_{\rho}\left(\left|D_{m_{j}, 0}\right|-\left|D_{m_{j}, e A_{\varepsilon, y}}\right|\right) G_{\rho},
$$

en changeant $x$ en $x+y$, puis $y$ en $y / \varepsilon$. Le potentiel magnétique $A_{\varepsilon, y}$ vaut alors

$$
A_{\varepsilon, y}(x):=\frac{1}{\varepsilon} A(\varepsilon x+y) .
$$

Cette quantité est certes localisée autour du vecteur $y$, mais demeure divergente dans la limite $\varepsilon \rightarrow 0$.

Afin d'éliminer cette divergence, nous faisons appel aux invariances physiques de la densité que nous étudions, à savoir les invariances par conjugaison de la charge et par changement de jauge. Ces invariances conduisent aux identités

$$
f_{\mathrm{vac}}^{\mathrm{PV}}\left(e \boldsymbol{A}_{\varepsilon}\right)(y)=f_{\mathrm{vac}}^{\mathrm{PV}}\left(-e \boldsymbol{A}_{\varepsilon}\right)(y)
$$

respectivement,

$$
f_{\mathrm{vac}}^{\mathrm{PV}}\left(e\left(\boldsymbol{A}_{\varepsilon}+\nabla \theta\right)\right)(y)=f_{\mathrm{vac}}^{\mathrm{PV}}\left(e \boldsymbol{A}_{\varepsilon}\right)(y),
$$

pour toute fonction $\theta \in \mathcal{C}^{2}\left(\mathbb{R}^{3}, \mathbb{R}\right)$.

Cette seconde identité conduit à une façon élémentaire d'éliminer la divergence de la formule (21) : il suffit de la concentrer dans un terme de type gradient. Cet argument est possible grâce à la formule suivante

$$
A_{\varepsilon, y}(x)=\nabla\left(x \cdot \int_{0}^{1} \frac{A(t \varepsilon x+y)}{\varepsilon} d t\right)+\frac{1}{2} B(y) \times x+\varepsilon R_{\varepsilon, y}(x),
$$

dans laquelle $B=\operatorname{rot} A$ et

$$
\varepsilon R_{\varepsilon, y}(x)=x \times \int_{0}^{1}(B(y)-B(t \epsilon x+y)) t d t .
$$


Notons que cette formule correspond à un passage à la jauge de Poincaré, puisque nous avons l'identité

$$
x \cdot\left(\frac{1}{2} B(y) \times x+\varepsilon R_{\varepsilon, y}(x)\right)=0 .
$$

Les invariances par conjugaison de la charge et par changement de jauge conduisent ainsi à l'expression

$$
\varepsilon^{3} f_{\mathrm{vac}}^{\mathrm{PV}}\left(e \boldsymbol{A}_{\varepsilon}\right)(y)=\frac{1}{2} \operatorname{tr} \sum_{j=0}^{2} c_{j} G_{\rho}\left(\left|D_{m_{j}, 0}\right|-\left|D_{m_{j}, \frac{e}{2} B(y) \wedge \cdot+\varepsilon e R_{\varepsilon, y}}\right|\right) G_{\rho},
$$

de laquelle toute divergence a disparu.

Nous sommes maintenant en position d'identifier la contribution principale à la limite que nous recherchons. Il s'agit de la quantité

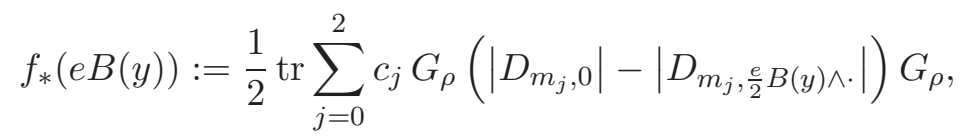

laquelle ne dépend que des opérateurs de Pauli à champ magnétique constant $B(y)$

$$
\pi_{B(y)}:=\left(\boldsymbol{\sigma} \cdot\left(-i \nabla-\frac{1}{2} B(y) \wedge \cdot\right)\right)^{2} .
$$

Afin de calculer cette contribution, nous commençons par rappeler les propriétés spectrales de ces opérateurs.

Donnons-nous un champ magnétique constant $B$, non nul et orienté suivant le vecteur $e_{3}=(0,0,1)$, soit de la forme $B=b e_{3}$, avec $b>0$. L'opérateur de Pauli $\pi_{B}$ se décompose alors sous la forme

$$
\pi_{B}:=\pi_{B}^{\perp}-\partial_{3}^{2} .
$$

Dans cette expression, l'opérateur de Pauli deux-dimensionnel $\pi_{B}^{\perp}$ est égal à

$$
\pi_{B}^{\perp}=-\Delta^{\perp}-b x^{\perp} \wedge\left(-i \nabla^{\perp}\right)+\frac{b^{2}}{4}\left|x^{\perp}\right|^{2}-b \sigma_{3},
$$

avec $x^{\perp}:=\left(x_{1}, x_{2}\right), \nabla^{\perp}:=\left(\partial_{1}, \partial_{2}\right)$ et $\Delta^{\perp}:=\partial_{1}^{2}+\partial_{2}^{2}$. L'opérateur $\pi_{B}^{\perp}$ est relié à l'oscillateur harmonique. Son spectre est bien connu [24]. Il est constitué des niveaux de Landau $2 b m$, où $m \in \mathbb{N}$. Le noyau intégral du projecteur spectral $P_{2 b m}\left(\pi_{B}^{\perp}\right)$ sur le niveau de Landau $2 b m$ est de plus explicite. Il est égal à

$$
\begin{aligned}
P_{2 b m}\left(x^{\perp}, y^{\perp}\right)=\frac{b}{2 \pi} e^{-\frac{i b}{2} x^{\perp} \wedge y^{\perp}} e^{-\frac{b}{4}\left|x^{\perp}-y^{\perp}\right|^{2} \times} & \\
& \times\left(\begin{array}{cc}
L_{m}\left(\frac{b}{2}\left|x^{\perp}-y^{\perp}\right|^{2}\right) & 0 \\
0 & \delta_{m \neq 0} L_{m-1}\left(\frac{b}{2}\left|x^{\perp}-y^{\perp}\right|^{2}\right)
\end{array}\right),
\end{aligned}
$$

où la notation $L_{m}$ désigne le polynôme de Laguerre d'ordre $m$.

Il s'ensuit une diagonalisation explicite de l'opérateur $\pi_{B}^{\perp}$ suivant la formule

$$
\pi_{B}^{\perp}=\sum_{m=0}^{\infty} 2 b m P_{2 b m}\left(\pi_{B}^{\perp}\right) .
$$


Afin d'étendre cette diagonalisation à l'opérateur de Pauli $\pi_{B}$, il reste à diagonaliser l'opérateur $-\partial_{3}^{2}$ grâce à la transformation de Fourier. Cette étude spectrale s'étend à un champ magnétique $B$ arbitraire grâce à l'invariance par rotation des quantités étudiées.

Nous appliquons cette étude au calcul de la contribution principale $f_{*}(e B(y))$. Cette densité est égale à

$$
f_{*}(e B(y))=\frac{1}{8 \pi^{2}} \int_{0}^{\infty} d s s^{-3}\left(\sum_{j=0}^{2} c_{j} e^{-s m_{j}^{2}}\right)(e s|B(y)| \operatorname{coth}(e s|B(y)|)-1) .
$$

C'est exactement la densité de l'énergie d'Euler-Heisenberg renormalisée $f_{\text {vac }}^{\mathrm{rEH}}(e B(y))$.

Pour un champ magnétique d'orientation constante $B(y)=b(y) e_{3}$, avec $b(y)>0$, l'analyse précédente revient à l'introduction d'opérateurs cohérents magnétiques $\Pi(m, p, y)$ sur l'espace $L^{2}\left(\mathbb{R}^{3}, \mathbb{C}^{2}\right)$, de noyau intégral

$$
\Pi(m, p, y)\left(x, x^{\prime}\right)=G_{\rho}(x-y) e^{i p x_{3}} P_{2 b(y) m}\left(x^{\perp}, x^{\perp}\right) e^{-i p x_{3}^{\prime}} G_{\rho}\left(x^{\prime}-y\right),
$$

pour tout $\left(x, x^{\prime}\right) \in \mathbb{R}^{6}$. Ces opérateurs satisfont la partition de l'identité

$$
\frac{1}{2 \pi} \sum_{m=0}^{+\infty} \int_{\mathbb{R}^{3}} \int_{\mathbb{R}^{3}} \Pi(m, p, y) d p d y=\mathbb{1}_{L^{2}\left(\mathbb{R}^{3}, \mathbb{C}^{2}\right)},
$$

sur laquelle repose le calcul de la contribution principale $f_{*}(e B(y))$. Nous avons utilisé cette partition en deux temps : une phase de localisation, puis une phase de projection sur les espaces propres de l'opérateur de Pauli $\pi_{B(y)}$. Il est ainsi plus facile de traiter l'orientation, en pratique non constante, du champ magnétique $B(y)$.

Afin de conclure la preuve du théorème 3 , il nous faut contrôler les termes de reste de la différence

$$
\begin{aligned}
& r\left(e \boldsymbol{A}_{\varepsilon}\right)(y):=\varepsilon^{3} f_{\mathrm{vac}}^{\mathrm{PV}}\left(e \boldsymbol{A}_{\varepsilon}\right)(y)-f_{\mathrm{vac}}^{\mathrm{rEH}}(e B(y)) \\
& =\frac{1}{2} \operatorname{tr} \sum_{j=0}^{2} c_{j} G_{\rho}\left(\left|D_{m_{j}, \frac{e}{2} B(y) \wedge \cdot \mid}\right|-\left|D_{m_{j}, \frac{e}{2} B(y) \wedge \cdot+\varepsilon e R_{\varepsilon, y}}\right|\right) G_{\rho} .
\end{aligned}
$$

Nous utilisons d'abord la version fonctionnelle de la formule

$$
|x|=\frac{1}{\pi} \int_{\mathbb{R}} \frac{x^{2}}{x^{2}+\omega^{2}} d \omega
$$

afin d'écrire le reste $r\left(e \boldsymbol{A}_{\varepsilon}\right)(y)$ sous la forme

$$
r\left(e \boldsymbol{A}_{\varepsilon}\right)(y)=\frac{1}{\pi} \int_{\mathbb{R}} \operatorname{tr} \sum_{j=0}^{2} c_{j} G_{\rho}\left(\frac{1}{\pi_{B(y)}+m_{j}^{2}+\omega^{2}}-\frac{1}{\pi_{B(y)}+m_{j}^{2}+\omega^{2}+\varepsilon S(y)}\right) G_{\rho} \omega^{2} d \omega .
$$

Les opérateurs $S(y)$ sont ici donnés par l'expression

$$
S(y):=e\left\{i \nabla, R_{\varepsilon, y}\right\}+e^{2} B(y) \wedge x \cdot R_{\varepsilon, y}+e^{2} \varepsilon\left|R_{\varepsilon, y}\right|^{2}-e \operatorname{rot} R_{\varepsilon, y} \cdot \boldsymbol{\sigma} .
$$

Nous développons ensuite ce reste de façon géométrique suivant l'identité

$$
\begin{aligned}
\frac{1}{T_{j}(\omega, y)}-\frac{1}{T_{j}(\omega, y)+\varepsilon S(y)}= & -\sum_{k=1}^{5}(-\varepsilon)^{k} \frac{1}{T_{j}(\omega, y)}\left(S(y) \frac{1}{T_{j}(\omega, y)}\right)^{k} \\
& -\varepsilon^{6}\left(\frac{1}{T_{j}(\omega, y)} S(y)\right)^{3} \frac{1}{T_{j}(\omega, y)+\varepsilon S(y)}\left(S(y) \frac{1}{T_{j}(\omega, y)}\right)^{3}
\end{aligned}
$$


dans laquelle nous avons noté

$$
T_{j}(\omega, y):=\pi_{B(y)}+m_{j}^{2}+\omega^{2} .
$$

Ceci conduit à l'expression

$$
r\left(e \boldsymbol{A}_{\varepsilon}\right)(y)=\sum_{k=1}^{5} r_{k}\left(e \boldsymbol{A}_{\varepsilon}\right)(y)+r_{6}^{\prime}\left(e \boldsymbol{A}_{\varepsilon}\right)(y),
$$

avec

$$
r_{k}\left(e \boldsymbol{A}_{\varepsilon}\right)(y):=\frac{(-1)^{k-1} \varepsilon^{k}}{2} \operatorname{tr} \sum_{j=0}^{2} c_{j} G_{\rho} \frac{1}{T_{j}(\omega, y)}\left(S(y) \frac{1}{T_{j}(\omega, y)}\right)^{k} G_{\rho},
$$

pour $1 \leq k \leq 5$, et

$$
r_{6}^{\prime}\left(e \boldsymbol{A}_{\varepsilon}\right)(y):=-\frac{\varepsilon^{6}}{2} \operatorname{tr} \sum_{j=0}^{2} c_{j} G_{\rho}\left(\frac{1}{T_{j}(\omega, y)} S(y)\right)^{3} \frac{1}{T_{j}(\omega, y)+\varepsilon S(y)}\left(S(y) \frac{1}{T_{j}(\omega, y)}\right)^{3} G_{\rho} .
$$

L'avantage de ce développement réside dans le fait que les opérateurs $1 / T_{j}(\omega, y)$ sont des résolvantes de l'opérateur de Pauli $\pi_{B(y)}$. Grâce à la formule (22), les noyaux intégraux de ces résolvantes sont explicites. Pour un champ magnétique de la forme $B(y)=b(y) e_{3}$, avec $b(y)>0$, ils sont donnés par la formule intégrale

$$
\begin{aligned}
\frac{1}{T_{j}(\omega, y)}\left(x, x^{\prime}\right)=\frac{b(y)}{(4 \pi)^{\frac{3}{2}}} e^{-\frac{i b(y)}{2} x^{\perp} \wedge x^{\perp \perp}} \int_{0}^{\infty} \frac{d t}{t^{\frac{1}{2}} \sinh (b(y) t)} \times \\
\quad \times e^{-\left(m_{j}^{2}+\omega^{2}\right) t-\frac{b(y)}{4} \operatorname{coth}(b(y) t)\left|x^{\perp}-x^{\perp \perp}\right|^{2}-\frac{1}{4 t}\left(x_{3}-x_{3}^{\prime}\right)^{2}}\left(\begin{array}{cc}
e^{b(y) t} & 0 \\
0 & e^{-b(y) t}
\end{array}\right),
\end{aligned}
$$

pour tout $\left(x, x^{\prime}\right) \in \mathbb{R}^{6}$. Nous pouvons estimer les normes $L^{p}$ de ces noyaux intégraux, et combiner ces estimations avec les propriétés d'intégrabilité du champ magnétique $B$, et de son gradient $\nabla B$, pour contrôler les termes de reste $r_{k}\left(e \boldsymbol{A}_{\varepsilon}\right)(y)$ et $r_{6}^{\prime}\left(e \boldsymbol{A}_{\varepsilon}\right)(y)$. Cette stratégie conduit à l'estimation finale

$$
\varepsilon^{3} \int_{\mathbb{R}^{3}} f_{\mathrm{vac}}^{\mathrm{PV}}\left(e \boldsymbol{A}_{\varepsilon}\right)(y) d y-\int_{\mathbb{R}^{3}} f_{*}(e B(y)) d y=\int_{\mathbb{R}^{3}} r\left(e \boldsymbol{A}_{\varepsilon}\right)(y) d y=\underset{\varepsilon \rightarrow 0}{\mathcal{O}}(\varepsilon),
$$

qui est exactement celle du théorème 3. Nous renvoyons à [18] pour de plus amples détails quant aux aspects techniques de la preuve de ce théorème.

\section{Références}

[1] V. Bach, E.-H. Lieb, and J.-P. Solovej. Generalized Hartree-Fock theory and the Hubbard model. J. Stat. Phys., 76(1-2) :3-89, 1994.

[2] M.G. Baring and A.K. Harding. Photon splitting and pair creation in highly magnetized pulsars. Astrophys. J., 547(2) :929-948, 2001.

[3] D.L. Burke, R.C. Field, G. Horton-Smith, J.E. Spencer, D. Walz, S.C. Berridge, W.M. Bugg, K. Shmakov, A.W. Weidemann, C. Bula, K.T. McDonald, E.J. Prebys, C. Bamber, S.J. Boege, T. Koffas, T. Kotseroglou, A.C. Melissinos, D.D. Meyerhofer, D.A. Reis, and W. Ragg. Positron production in multiphoton light-by-light scattering. Phys. Rev. Lett., 79(9) :1626-1629, 1997. 
[4] H.B.G. Casimir. On the attraction between two perfectly conducting plates. Proc. Kon. Nederland. Akad. Wetensch., 51(7) :793-795, 1948.

[5] H.B.G. Casimir and D. Polder. The influence of retardation on the London-van der Waals forces. Phys. Rev., 73(4) :360-372, 1948.

[6] S. Chadha and P. Olesen. On Borel singularities in quantum field theory. Phys. Lett. B, 72(1) :87-90, 1977.

[7] V.I. Denisov and S.I. Svertilov. Vacuum nonlinear electrodynamics curvature of photon trajectories in pulsars and magnetars. A\&3A, 399(3) :L39-L42, 2003.

[8] P.A.M. Dirac. Theory of electrons and positrons. Nobel lecture delivered at Stockholm, 1933.

[9] P.A.M. Dirac. Discussion of the infinite distribution of electrons in the theory of the positron. Proc. Camb. Philos. Soc., 30 :150-163, 1934.

[10] P.A.M. Dirac. Théorie du positron. In Structure et propriétés des noyaux atomiques, number XXV in 7th Solvay Report, pages 203-212. Gauthier-Villars, Paris, 1934.

[11] L. Erdös and J.-P. Solovej. Semiclassical eigenvalue estimates for the Pauli operator with strong non-homogeneous magnetic fields. II. Leading order asymptotic estimates. Commun. Math. Phys., 188(3) :599-656, 1997.

[12] L. Erdös and J.-P. Solovej. Semiclassical eigenvalue estimates for the Pauli operator with strong nonhomogeneous magnetic fields. I. Nonasymptotic Lieb-Thirring-type estimate. Duke Math. J., 96(1) :127-173, 1999.

[13] L. Erdös and J.-P. Solovej. Uniform Lieb-Thirring inequality for the three-dimensional Pauli operator with a strong non-homogeneous magnetic field. Ann. Henri Poincaré, $5(4): 671-741,2004$.

[14] H. Euler. Über die Streuung von Licht an Licht nach der Diracschen Theorie. Ann. d. Phys., 418(5) :398-448, 1936.

[15] H. Euler and B. Kockel. Über die Streuung von Licht an Licht nach der Diracschen Theorie. Naturwissenschaften, 23(15) :246-247, 1935.

[16] P. Gravejat, C. Hainzl, M. Lewin, and É. Séré. Two Hartree-Fock models for the vacuum polarization. In Journées Équations aux Dérivées Partielles, 2012. Exp. No. IV, $31 \mathrm{p}$.

[17] P. Gravejat, C. Hainzl, M. Lewin, and É. Séré. Construction of the Pauli-Villarsregulated Dirac vacuum in electromagnetic fields. Arch. Ration. Mech. Anal., 208(2) :603-665, 2013.

[18] P. Gravejat, M. Lewin, and É. Séré. Derivation of the magnetic Euler-Heisenberg energy. Preprint, 2016. https://hal.archives-ouvertes.fr/hal-01270637v2.

[19] W. Greiner and J. Reinhardt. Quantum electrodynamics. Springer-Verlag, Berlin, Fourth edition, 2009. Translated from the German.

[20] G.'t Hooft and M. Veltman. Regularization and renormalization of gauge fields. $\mathrm{Nu}$ clear Physics B, 44(1) :189-213, 1972.

[21] C. Hainzl, M. Lewin, and J.-P. Solovej. The mean-field approximation in quantum electrodynamics. The no-photon case. Commun. Pure Appl. Math., 60(4) :546-596, 2007.

[22] W. Heisenberg and H. Euler. Folgerungen aus der Diracschen Theorie des Positrons. Zts. f. Phys., 98(11-12): 714-732, 1936.

[23] W.E. Lamb and R.C. Retherford. Fine structure of the hydrogen atom by a microwave method. Phys. Rev., 72(3) :241-243, 1947. 
[24] L.D. Landau and E.M. Lifshitz. Quantum mechanics. Non-relativistic theory, volume 3 of Course of Theoretical Physics. Pergamon Press, Oxford, Third edition, 1977. Translated from the Russian by J.B. Sykes and J.S. Bell.

[25] M. Lewin. A nonlinear variational problem in relativistic quantum mechanics. In R. Latala, A. Rucinski, P. Strzelecki, J. Swiatkowski, D. Wrzosek, and P. Zakrzewski, editors, Proceedings of the sixth European congress of mathematics, Krakow (Poland), July 2012, pages 45-59. European Mathematical Society, 2014.

[26] E.H. Lieb, J.-P. Solovej, and J. Yngvason. Asymptotics of heavy atoms in high magnetic fields. II. Semiclassical regions. Commun. Math. Phys., 161(1) :77-124, 1994.

[27] M. Marklund, G. Brodin, and L. Stenflo. Electromagnetic wave collapse in a radiation background. Phys. Rev. Let., 91(16) :163601, 2003.

[28] G.A. Mourou, T. Tajima, and S.V. Bulanov. Optics in the relativistic regime. Rev. Mod. Phys., 78(2) :309-371, 2006.

[29] W. Pauli and F. Villars. On the invariant regularization in relativistic quantum theory. Rev. Modern Phys., 21 :434-444, 1949.

[30] M.E. Peskin and D.V. Schroeder. An introduction to quantum field theory, volume 94 of Frontiers in Physics. Westview Press, New-York, 1995.

[31] J. Schwinger. On gauge invariance and vacuum polarization. Phys. Rev., 82(5) :664$679,1951$.

[32] E.A. Uehling. Polarization effects in the positron theory. Phys. Rev., 48(1) :55-63, 1935.

[33] V.F. Weisskopf. Über die Elektrodynamik des Vakuums auf Grund der Quantentheorie des Elektrons. Dan. Mat. Fys. Medd., 16(6) :1-39, 1936.

[34] K.G. Wilson. Confinement of quarks. Phys. Rev. D, 10(8) :2445-2459, 1974.

[35] J. Yngvason. Thomas-Fermi theory for matter in a magnetic field as a limit of quantum mechanics. Lett. Math. Phys., 22(2) :107-117, 1991. 